\title{
Heterosis Relating to a Terminal Inversion in Artificial Population of Drosophila ananassae
}

\author{
Yoshiko N. ToBarI \\ Department of Biology, Faculty of Science, Tokyo Metropolitan University
}

Received April 19, 1962

It is well known that a chromosomal inversion, when it occurs, prevents crossing over of gene blocks included within it. Accordingly, two reciprocal types of chromosomal arrangements resulting from an inversion should act as a pair of allelic genes. In fact, with some of these chromosomal variants in Drosophila populations, balanced chromosomal polymorphisms have often been established by "heterosis", where structural heterozygotes are more adaptive than homozygotes.

In Drosophila ananassae, when two gene arrangements by a subterminal inversion in the left arm of the second chromosome were mixed in a population cage, their relative frequencies always attained a constant equilibrium value as the result of heterosis (Moriwaki et al. 1955). On the other hand, in the same species, another inversion, In $3 \mathrm{~L}$, a terminal inversion in the left arm of the third chromosome has long been known and it constitutes a suggestive case where the inversion may not suppress crossing over (Kikkawa 1938; Moriwaki 1950).

In the present experiments, this inversion, In $3 \mathrm{~L}$, was studied to examine if it could establish a balanced chromosomal polymorphism. Clarification of the following three major points were attempted.

1) Success or failure of establishment of balanced chromosomal polymorphism regarding this terminal inversion in population cages.

2) Comparison of phenotypic differences among the karyotypes, with respect to each life cycle component.

3) Estimation of relative adaptive values of gene arrangements on the basis of the components of the life cycle, and comparison of them with those computed by population cage technique.

\section{Materials and Methods}

The two reciprocal gene arrangements, In3L-A and -B, were respectively fixed as homozygotes, isolated from "Hawaii" strain of Drosophila ananassae, which was sent by the University of Texas and has been kept in our laboratory since 1950. Four cages were set up in which In3L-A and -B were mixed with different initial frequencies. The length of generation was estimated as 15 days. The bottom of the cage had 15 holes for insertion of food cups. 14 cups were used for feeding, among which, since 
2 cups with fresh medium were inserted every third day, each cup remained in the cage for a period of 21 days. The central cup was used for obtaining samples. Sampling was performed once a month for first several months and once in 2 months thereafter. One sample consisted of 150 larvae collected on 6 successive days, 25 larvae for each day. The eggs were allowed to develop at optimal condition in culture bottles and 150 of the larvae were used for microscopic examination of the salivary gland chromosomes.

For the analysis of heterosis, the examination of life cycle components was made. The following experimental procedure was employed in order to minimize the effect of inbreeding and obtain flies possibly isogenic for the genetic background other than A- or B-type of the third chromosome. Ten strains of A-homozygotes and 10 of Bhomozygotes were isolated at random from a population cage in which an equilibrium between In3L-A and -B has been established. Then equal number of virgin females and males from each strain were collected and grouped to be used for crosses as follows:

$\mathrm{AA}$ 우우 (from 10 strains) $\times \mathrm{BB}$ 令 (from 10 strains)— $\mathrm{AB}$ heterozygotes

$\mathrm{BB}$ 우우 (from 10 strains) $\times \mathrm{AA} \hat{\delta} \hat{\delta}$ (from 10 strains)—BA heterozygotes

$\mathrm{AA}$ 우우 (from 10 strains) $\times \mathrm{AA}$ 令令 (from 10 strains)—AA homozygotes

$\mathrm{BB}$ 우우 (from 10 strains) $\times \mathrm{BB}$ 옹 (from 10 strains) - $\mathrm{BB}$ homozygotes

From these crosses, 4 types of eggs and adults were obtained, the formers were to be tested for hatchability, developmental rate and viability and the latters to be tested for longevity and fecundity. Throughout the experiment temperature was kept at $25^{\circ} \mathrm{C}$.

\section{Experimental Results}

\section{Artificial population studies}

Four population cages, $\mathrm{C}-2, \mathrm{C}-1, \mathrm{C}-5$ and $\mathrm{C}-3$ were initiated with about $90 \% \mathrm{~A}$ and $10 \% \mathrm{~B}, 70 \% \mathrm{~A}$ and $30 \% \mathrm{~B}, 50 \% \mathrm{~A}$ and $50 \% \mathrm{~B}, 30 \% \mathrm{~A}$ and $70 \% \mathrm{~B}$, respectively.

Fig. 1 shows the changes observed in these populations. All 4 populations reached equilibria at about $60 \% \mathrm{~A}$ and $40 \% \mathrm{~B}$ after 3 4 months regardless of the initial frequencies of the chromosome types. Accordingly it is evident that balanced chromosomal polymorphism was established in the populations. Relative adaptive values $(\mathrm{W})$ and selection coefficients ( $s$ and $t$ ) of the karyotypes were calculated according to the method adopted by Wright and Dobzhansky (1946) and modified somewhat by Yoshida (unpub.). The values obtained were as follows:

$\begin{array}{cccc} & \text { Genotype } & \text { W } & \\ \text { C }-2 & \text { AA } & 0.50 & s=0.50\end{array} \quad \hat{q}_{A}=0.60$




$\begin{array}{lllll} & \mathrm{AA} & 0.73 & s=0.27 & \hat{q}_{A}=0.57 \\ \mathrm{C}-1 & \mathrm{AB} & 1.00 & t=0.36 & \\ & \mathrm{BB} & 0.64 & & \\ \mathrm{C}-5 & \mathrm{AA} & 0.78 & s=0.22 & \hat{q}_{A}=0.61 \\ & \mathrm{AB} & 1.00 & t=0.35 & \\ & \mathrm{BB} & 0.65 & & \\ \mathrm{C}-3 & \mathrm{AA} & 0.67 & s=0.33 & \hat{q}_{A}=0.62 \\ & \mathrm{AB} & 1.00 & t=0.54 & \\ & \mathrm{BB} & 0.46 & & \end{array}$

\section{Analysis of heterosis}

In these experiments, the physiological traits seemingly related to heterosis were examined, making the cultural conditions rather unfavorable approximating cage conditions. For the fecundity and the longevity experiments, mass matings of 35

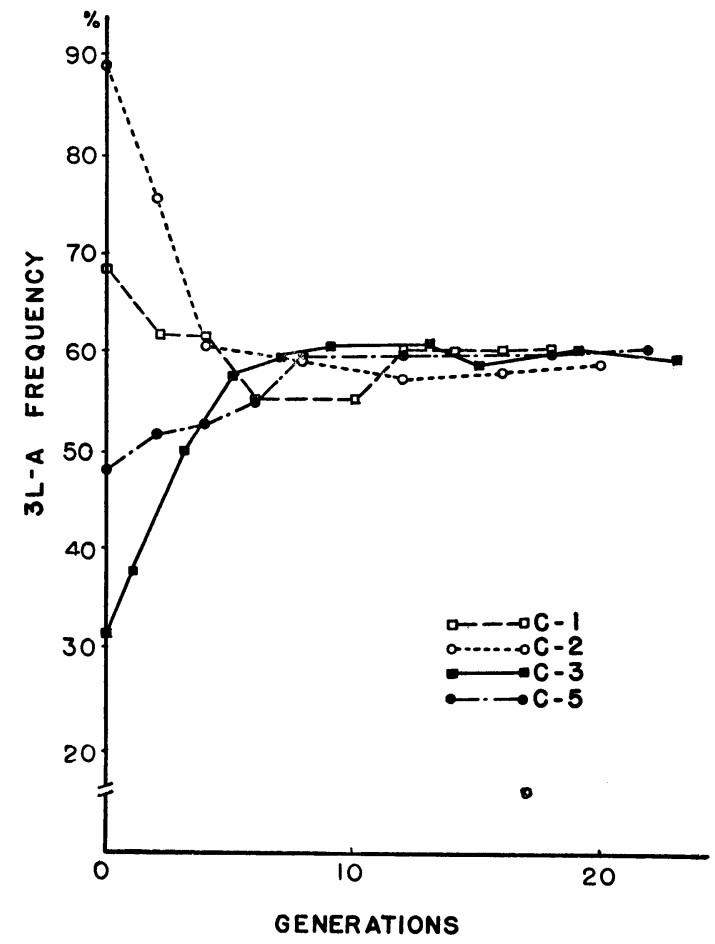

Fig. 1. Changes observed in frequencies of In3L-A in artificial populations containing In $3 \mathrm{~L}-\mathrm{A}$ and -B arrangements. flies of each sex were made in $2.5 \times 12$ $\mathrm{cm}$ vials with ordinary medium (lightly yeasted cornmeal-molasses-agar medium). Since the females were allowed to lay eggs for 24 hours, the medium became crowded with the larvae. Adult flies emerged from these vials were used.

(a) Fecundity: 10 newly-hatched females of one chromosomal type, and 20 males of the same type were placed together in a separate vial with ordinary medium. Eight bottles for each chromosomal type, in this manner, were set up and stored in a constant temperature room at $25^{\circ} \mathrm{C}$. Every day after transferrence of the flies to new vials, eggs laid on the medium were counted. Only for first 2 weeks, during which high fecundity lasted, records were continually taken. The results were summarized in Table 1. Heterosis was seen in $\mathrm{AB}$ heterozygote showing the highest number of eggs per 10 females per day, while BA heterozygote showed the lower fecundity than $\mathrm{AB}$, although no less than homozygotes. 
Table 1. Egg production at $25^{\circ} \mathrm{C}$

\begin{tabular}{c|c|c}
\hline Type & $\begin{array}{c}\text { No. of eggs/10 } \\
\text { females/day }\end{array}$ & Relative fecundity \\
\hline AA & $57.7 \pm 6.2$ & 0.893 \\
AB & $68.8 \pm 9.2$ & 1.000 \\
BA & $44.2 \pm 6.2$ & 0.642 \\
BB & $44.7 \pm 7.2$ & 0.650 \\
\hline
\end{tabular}

Significant differences: $A B>B B, A B>B A$

Table 2. Mean longevities and relative longevities of different genotypes at $25^{\circ} \mathrm{C}$

\begin{tabular}{c|c|c}
\hline \hline Type & $\begin{array}{c}\text { Mean longevity } \\
\text { in days }\end{array}$ & Relative longevity \\
\hline $\mathrm{AA}$ & $28.12 \pm 0.78$ & 0.919 \\
$\mathrm{AB}$ & $30.64 \pm 0.82$ & 1.000 \\
$\mathrm{BA}$ & $33.92 \pm 0.92$ & 1.107 \\
$\mathrm{BB}$ & $23.72 \pm 0.42$ & 0.774 \\
\hline
\end{tabular}

Significant differences: $B A>A B>A A>B B$

(b) Longevity of adults: 20 females and 20 males of newly emerged flies from crowded larval conditions to be tested were placed in the regular culture bottles with ordinary medium. Eight bottles for each karyotype were set up at the same time. Everyday the survivors of each bottle were transferred to a fresh bottle and the number of deaths occurring for one day was recorded. Heterosis is obvious in this trait. The results showing that both heterozygotes $\mathrm{AB}$ and $\mathrm{BA}$ have significantly greater mean longevities than both homozygotes are given in Table 2.

(c) Hatchability of eggs: The parent flies (30 females and 30 males) aged about 5 7 days, having high egg laying capacity, were introduced into separate vials with the test medium for hatchability and allowed to lay eggs for 2 hours. After the parent flies were discarded, eggs laid on food were counted under a binocular microscope. About 100 eggs per vial randomly selected were left to be examined for hatchability and others were removed. The vials were stored at $25^{\circ} \mathrm{C}$ for 40 hours. Thereafter the newly hatched larvae and unhatched eggs in each vial were counted. Eight bottles for each type were run at the same time and the results of this experiment were summarized in Table 3. The percentage of hatched larvae in both heterozygotes was significantly higher than that of $\mathrm{BB}$ homozygote, but not significantly so than that of AA homozygote. 
Table 3. Hatchability of eggs at $25^{\circ} \mathrm{C}$

\begin{tabular}{c|c|c|c|c}
\hline $\begin{array}{c}\text { Genotype } \\
\text { of eggs }\end{array}$ & Eggs tested & Eggs hatched & $\begin{array}{c}\text { Percent } \\
\text { hatched }\end{array}$ & $\begin{array}{c}\text { Relative } \\
\text { hatchability }\end{array}$ \\
\hline AA & 819 & 532 & 65.0 & 0.888 \\
AB & 756 & 555 & 73.4 & 1.000 \\
BA & 786 & 528 & 67.2 & 0.915 \\
BB & 820 & 498 & 60.7 & 0.829 \\
\hline
\end{tabular}

Significant differences: $A B>B B, B A>B B$

Table 4. Viabilities and relative viabilities of different genotypes at $25^{\circ} \mathrm{C}$

\begin{tabular}{c|c|c|c|c}
\hline Type & $\begin{array}{c}\text { No. of 1st } \\
\text { inst. larvae }\end{array}$ & $\begin{array}{c}\text { No. of adults } \\
\text { emerged }\end{array}$ & $\begin{array}{c}\text { Percent } \\
\text { emerged }\end{array}$ & $\begin{array}{c}\text { Relative } \\
\text { viability }\end{array}$ \\
\hline AA & 250 & 202 & 80.8 & 0.948 \\
AB & 250 & 213 & 85.2 & 1.000 \\
BA & 250 & 205 & 82.0 & 0.962 \\
BB & 250 & 153 & 61.2 & 0.718 \\
\hline
\end{tabular}

Significant differences: $\mathrm{AB}>\mathrm{BB}, \mathrm{BA}>\mathrm{BB}, \mathrm{AA}>\mathrm{BB}$

(d) Pre-adult viability: In the same way as the hatchability experiment, females were allowed to oviposit for 2 hours on sugar-agar medium in separate vials. After these vials were kept for 24 hours at $25^{\circ} \mathrm{C}$, newly hatched first instars were collected and 50 of them belonging to one karyotype were placed on the food $(4 \mathrm{cc}$ of the $5 \%$ boiled yeast-sugar-agar medium) which was not sufficient to support 50 larvae, approximating the crowded condition in a cage to be compared later. Five bottles for each type were started simultaneously. Emerged flies from these vials were counted daily. The results are given in Table 4. From the percentage of eclosion BB homozygote showed significantly lower viability than the other three types, among which no significant differences could be detected.

(e) Rate of development: The experimental procedure was the same as before except for 2 nutritional conditions, namely, $20 \%$ boiled-yeast medium as a favorable one and 5\% boiled-yeast medium as poor one. About 100 eggs among those laid during 2 hours were left on each medium. Four bottles for each condition for one type were set up at the same time. Every 2 hours new pupae were counted. Table 5 gives the mean hours elapsed before pupation for each karyotype. In both conditions the heterozygotes $\mathrm{AB}$ and $\mathrm{BA}$ developed faster than the homozygotes, showing obvious heterosis. In $20 \%$ yeast no differences could be found between the homozygotes as well as between the heterozygotes, while in $5 \%$ yeast significant differences were 
obtained in the direction that $\mathrm{AA}$ was faster than $\mathrm{BB}$, and $\mathrm{AB}$ was faster than $\mathrm{BA}$.

Table 5. Rate of development: Duration of larval life in hours at $25^{\circ} \mathrm{C}$

\begin{tabular}{c|c|c|c}
\hline \hline Type & $20 \%$ yeast & $5 \%$ yeast & $\begin{array}{c}\text { Relative rate of develop- } \\
\text { ment (in 5\% yeast) }\end{array}$ \\
\hline $\mathrm{AA}$ & $109.74 \pm 0.20$ & $180.16 \pm 1.32$ & 0.852 \\
$\mathrm{AB}$ & $107.89 \pm 0.20$ & $153.64 \pm 2.61$ & 1.000 \\
$\mathrm{BA}$ & $108.58 \pm 0.21$ & $163.96 \pm 1.33$ & 0.937 \\
$\mathrm{BB}$ & $110.02 \pm 0.19$ & $184.00 \pm 2.23$ & 0.835 \\
\hline
\end{tabular}

Significant differences: $A B>A A, A B>B B, B A>A A, B A>B B$ in $20 \%$ yeast, $\mathrm{AB}>\mathrm{BA}>\mathrm{AA}>\mathrm{BB}$ in $5 \%$ yeast

Table 6. Relative adaptive values of genotypes determined from life cycle components of flies raised from unfavorable condition at $25^{\circ} \mathrm{C}$

\begin{tabular}{|c|c|c|c|c|}
\hline Life cycle components & AA & $\mathrm{AB}$ & $\mathrm{BA}$ & $\mathrm{BB}$ \\
\hline Viability & .948 & 1 & .962 & .718 \\
\hline Longevity & .919 & 1 & 1.107 & .774 \\
\hline Fecundity & .893 & 1 & .642 & .650 \\
\hline Rate of development & .852 & 1 & .937 & .835 \\
\hline Egg hatchability & .888 & 1 & .915 & .829 \\
\hline Net $\mathrm{W}$ & .553 & 1 & .587 & .250 \\
\hline Net $W^{\prime}$ & .603 & \multicolumn{2}{|c|}{1} & .273 \\
\hline $\mathrm{W}$ (from cage experiments) & .69 & \multicolumn{2}{|c|}{1} & .55 \\
\hline
\end{tabular}

\section{Estimation of adaptive values}

An attempt was made by Wallace (1948) and Moos (1955) in D. pseudoobscura, and Spiess et al. (1956) in D. persimilis to estimate the combined effects of the above examined traits. Spiess wrote, "Granted that there are other factors in the fly life cycle which have yet to be tested, it is interest to compare the net fitness of the three karyotypes obtained by the methods above with adaptive values calculated from population cage data."

A similar approach was performed in $D$. ananassae. The relative adaptive values calculated according to the method worked out by Wallace (1948) were summarized in Table 6 . The values in the third line from the bottom show the net adaptive values (W) estimated combining the five main factors studied. The final net values $\left(\mathrm{W}^{\prime}\right)$ were estimated on the basis of chromosomal frequencies in the experimental popu- 
lations at equilibrium with $60 \% \mathrm{~A}$ and $40 \% \mathrm{~B}$, in which $20 \%$ of the heterozygotes may be considered as derived from $B B$ mothers. By using the calculated frequencies of $\mathrm{AB}$ and $\mathrm{BA}$, the total adaptive value of the mixture of $\mathrm{AB}$ and $\mathrm{BA}$ was calculated. By putting this as 1 the values of $\mathrm{AA}$ and $\mathrm{BB}$ were adjusted which are the final values $\left(\mathrm{W}^{\prime}\right)$.

By comparing these final adaptive values (net $\mathrm{W}^{\prime}$ ) with those estimated by population cage experiments $(\mathrm{W})$, it can be said that the value for $\mathrm{BB}$ of net $\mathrm{W}^{\prime}$ is underestimated, while the value of AA turns out to agree fairly well.

\section{Conclusion}

In the experimental populations with terminal inversion, In3L, balanced chromosomal polymorphism was established. Hence it is likely that the inversion, notwithstanding it had been believed not to suppress crossing over, plays the role to prevent the breakdown of gene blocks tied together, acting as a super-gene. As a matter of fact, from the other points it seems that this terminal inversion might suppress crossing over after all (Moriwaki 1958). Therefore the present result in artificial populations seems to give another evidence in support of the above statement by Moriwaki.

The experimental populations always came to an equilibrium with about $60 \% \mathrm{~A}$ and $40 \% \mathrm{~B}$, regardless of the initial frequencies. However the relative adaptive values, from cage experiments, of three karyotypes regarding the inversion (two homozygotes and one heterozygote) are not necessarily the same in every population. Although the reason why these values vary among cages is still unknown, it seems that the difference could be caused by some differences of internal environments rather than external ones. Further discussion will be seen elsewhere.

Moreover, the net adaptive values estimated by measuring life cycle components were considerably close to those from the cage experiments, although the value for $\mathrm{BB}$ was relatively discrepant. These results, in general, suggest that most of the components-measured in these experiments should be the factors which contribute to the adaptive values of karyotypes. BB homozygote which had the lowest adaptive value as compared with the values of $A A$ and $A B$ in the cage experiments was always most inferior with regard to all life cycle components examined, while $A B$ heterozygote was most superior regarding the all components, showing the highest adaptive value.

\section{Summary}

1. Artificial populations containing reciprocal arrangements, $A$ and $B$, of the third chromosome due to a terminal inversion, In3L, of Drosophila ananassae were studied. All populations reached equilibria at about $60 \% \mathrm{~A}$ and $40 \% \mathrm{~B}$ after $3 \sim 4$ months.

2. Heterosis regarding the karyotypes, $A A, B B$ and $A B$, was expressed in longe- 
vity, viability, hatchability, fecundity and rate of development.

3. AA was always superior to $B B$ in longevity, viability and rate of development.

4. Some differences between $\mathrm{AB}$ and $\mathrm{BA}$ heterozygotes raised by reciprocal crosses were observed in fecundity, longevity and rate of development in poor medium with only $5 \%$ boiled yeast.

5. Comparing net adaptive values estimated by measuring life cycle components with those from population cage experiments, a close coincidence was ascertained for $\mathrm{AB}$ as well as for $\mathrm{AA}$, although an underestimation was found for BB homozygote,

\section{Acknowledgement}

The author wishes to express her gratitude to Professor D. Moriwaki for his guidance during the course of this investigation. She is indebted also to Professor K. Dan who has kindly read the manuscript.

\section{Literature Cited}

Kikkawa, H. 1938 Studies on the genetics and cytology of Drosophila ananassae. Genetica 20: $458-516$.

Moos, J. R. 1955 Comparative physiology of some chromosomal types in Drosophila pseudoobscura. Evolution 9: 141-151.

Moriwaki, D. 1950 An inversion not suppressing crossing over in Drosophila ananassae. (Japanese) Oguma Commemoration Volume on Cytology and Genetics: 71-75.

-, M. Shirai and Y. H. Yoshida 1955 Balanced polymorphism attained in some experimental populations of D. ananassae. D. I. S. 29: (Part 2) 143-144.

1958 Genetic studies in Drosophila ananassae. (Japanese) Jap. Jour. Genet. 33: 364-377.

Spiess, E. B. and B. R. J. Schuellein 1956 Chromosomal adaptive polymorphism in Drosophila persimilis, I. Life cycle components under near optimal conditions. Genetics 41: 501-516.

Wallace, B. 1948 Studies on "sex-ratio" in Drosophila pseudoobscura, I. Selection and "sex-ratio". Evolution 2: 189-217.

Wright, S. and Th. Dobzhansky 1946 Genetics of natural populations, XII. Experimental reproduction of some of the changes caused by natural selection in certain populations of Drosophila pseudoobscura. Genetics 31: 125-156. 